\title{
Automated ROI Detection in Left Hand X-ray Images using CNN and RNN
}

\author{
Youngbok $\mathrm{Cho}^{1 *}$ and Sunghee $\mathrm{Woo}^{2}$ \\ ${ }^{1}$ Department of Computer \& Information Security, Daejeon University, Daejeon \\ 34520, Korea \\ ${ }^{2}$ Department of Medical IT Engineering, Korea National University of \\ Transportation, 61, Daehak-ro, Yonggang-ro, Jeungpyeong-gun, 27909, \\ Republic of Korea \\ ybcho@dju.kr
}

\begin{abstract}
Automatic segmentation of the area of interest in medical image processing is a very important but difficult problem. Deep learning algorithms can help clinicians and radiologists determine diagnosis and treatment plans. We propose and evaluate a probabilistic approach for automated region of interest ROIs detection using convolutional neural networks (CNNs). The proposed algorithm is simple and can be divide into regions and features can be extracted for the divided regions. We also propose a preprocessing algorithm based on CNN and RNN to automatically classify ROIs that are finely adjusted through image standardization based on TW3. The result is 20\%-40\% more accurate than those obtained using the conventional method. In addition, input image sensitivity is approximately $40 \%$ greater and the specificity was equal to or greater than $96 \%$.
\end{abstract}

Keywords: Deep Learning, Image Segmentation, Edge Detection, Region Detection, Region of Interesting

\section{Introduction}

While many parts of the body (e.g., hand, foot, knee, elbow, guard and hip) have been studied to develop a method to provide an accurately assess development, Tough has also been investigated. A bone age assessment is a procedure used in pediatric radiology for both diagnostic and therapeutic investigations of endocrinology problems, children growth and genetic disorders. [1, 2]. In addition, automated computer-assisted detection systems are used in a wide variety of health care applications, and such systems can potentially reduce radiologist workloads significantly and serve as a first or second reader to improve disease assessment. Most recent studies have been based on image feature extraction from a region of interest (ROI) [3, 4]. In general, ROI feature extraction from radiology images is used to assess bone maturity.

Many methods to determine bone age have been proposed, and the most common are the Greulich and Pyle(GP) and Tanner-Whitehouse (TW) methods [5, 6, 7]. TW based methods, TW2 and TW3, analyze specific bones, and instead of the whole hand as in the GP method, whose 20 standard maturity varies according to age population, race and gender. In particular, TW methods take into account a set of specific regions of interest (ROIs) divided into epiphysis/metaphysis ROIs (EMROIs) and carpal ROIs (CROIs). However, different results are produced when these methods are used with a single X-ray image. Therefore, to provide accurate and consistent evaluation of bone maturity, an

Received (March 8, 2018), Review Result (May 23, 2018), Accepted (May 30, 2018)

${ }^{*}$ Corresponding Author 
automated bone age measurement system based on a deep $\mathrm{CNN}$ is required. We propose an algorithm that uses the TW3 method based on a deep learning CNN to improve the accuracy of bone age determination.

We learn the exact pattern by separating the growth plate from an X-ray image of the left hand and learn the pattern on the growth plate using the deep CNN [8, 9]. Herein, the goal is to demonstrate the benefits of a customized CNN algorithm to evaluate bone age utilizing image datasets of children and adolescents in Korea. We designed 14 hidden layers, inception layers, and spatial transformer layers for the CNN. To determine bone maturity accurately, the proposed method extracts areas of interest from an entire image to construct a training dataset and learns using the extracted data to produce results with reduced error rates. It is usually performed by comparing an X-ray of a child's left hand and wrist to an atlas of known bones or by analyzing the specific features of bones such as the ratio of width to height or the degree of overlap with other bones. We investigated the maturity of 13 wrist and hand bones using the TW3 method to determine the rate at which bone age is estimated and the rate at which childhood growth is compromised by adding them up. It is also used to measure the average bone age of children and adolescents in Korea.

\section{Related Works}

\subsection{Greulich and Pyle (GP) and Tanner-Whitehouse (TW) Methods}

GP [7, 8, 9] standards were developed between 1931 and 1942 from hand and wrist radiographic images of white, upper-middle class, male, and female children included in the Brush Foundation growth study. Therefore, the GP method is most commonly used in the United States of America. However, GP methods are also used in Korea. The standard templates developed for male children comprise 31 radiographic images covering the growth stages between 0 and 19 years. Also, whereas the standard templates for female children comprise 27 radiographic images covering the growth stages between 0 and 18 years of age [7]. Note that these templates were developed separately for female and male children because bone growth and development displays gender-related differences. The GP method compares template images with input images to identify similar templates. The comparison proceeds until radiography results that correspond to most of the radiography images in a given case are found. In this case, the nearest matching template is selected because it is typically difficult to find a $100 \%$ match.

The TW method [7, 10,11] assigns score to relevant bone through a detailed structural analysis and evaluates the bone maturity based on the sum of the scores. Thirteen bone areas are used in this bone maturity determination analysis, including weak bone, small bone (RUS), and male bones. Herein, each step is used as a criterion for each score based on gender. There are three versions of the TW method, i.e., TW1, TW2, and TW3 [11, 12, 13]. TW2 determines bone maturity based on eight maturity indicators for each bone in the hand and wrist and nine indicators for the radius. Note that the method used to calculate bone maturity is the same for TW1 and TW2, except for changes made to differentiate males and females. However, the reference standards are based on image data from the 1950s and 1960s. Thus, the scoring system, goal maturity reference image, and equation used to predict adult stature were modified to ensure accuracy and repeatable results. The 20-bone score used in the TW3 method for bone age assessment was abolished, and the reference values and charts were changed based on North America criteria. 


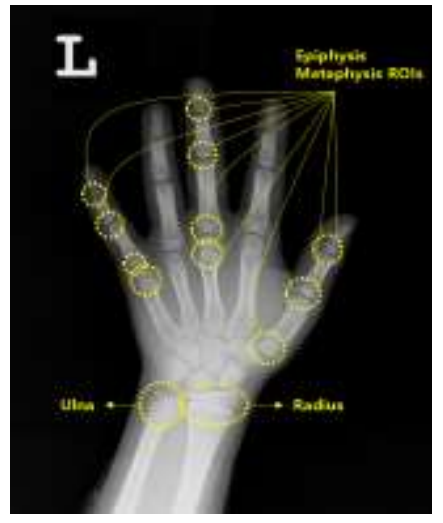

\section{Figure 1. Regions of Interest (ROIs) used in the Tanner Whitehouse method: Epiphysis/Metaphysis ROls and Carpal ROls}

With the GP mothed, a pediatrician examines hand bone radiology images and compares it to images in a hand bone radiology atlas that are assumed to be most similar to the target patient. In contrast, the TW3 mothed defines 20 ROIs to determine the age. Note that the TW3 mothed requires more time; however, it is more accurate than the GP method. The TW2 mothed uses a radius, ulnar (RUS), and wrist (Carpal) bones to determine bone maturity, while TW3 uses RUS and carpal bone to determine an independent score.

\subsection{Deep Convolution Neural Network}

Tremendous progress has been made in image recognition, primarily due to the availability of large-scale annotated datasets. CNNs have been applied to image recognition tasks since the late 1980s [14, 15]. Image classification, which can be defined as categorizing images into one of several predefined classes, is a fundamental problem in computer vision CNNs are a type of feedforward neural network in machine learning. A CNN is formed by a stack of layers $[12,13,16]$ or a directed acyclic graph of layers $[17,18,19]$. A CNN usually combines the following five types of layers. It forms the basis for other computer vision tasks such as localization, detection, and segmentation. CNNs are feedforward networks in that the information flow is unidirectional, i.e., from the input to output. There are various $\mathrm{CNN}$ architectures; however, CNN architectures generally comprise only convolutional and pooling layers grouped into modules, and one or more fully connected layer follow these modules (similar to a standard feedforward neural network). In addition, the modules are often stacked to from a deep model [20]. The convolutional layers serve as feature extractors; thus, they learn the feature representations of the input images, and the neurons in the convolutional layers are arranged into feature maps. All neurons within a feature map have weights that are constrained to be equal; however, different feature maps within the same convolutional layer have different weights such then several features can be extracted at each location. Formally, the $k^{\text {th }}$ output feature map $Y_{k}$ can be computed as $f(\square)$. The output of the pooling operation associated with the $k^{\text {th }}$ feature map is denoted by $Y_{k i j}$ :

$$
\begin{aligned}
& Y_{k}=f\left(W_{k} \times x\right) \\
& Y_{k i j}=\max _{(p, q) \in \square} x_{k j}
\end{aligned}
$$

Here, the input image is denoted $x$; the convolutional filter related to the $k^{\text {th }}$ feature map is denoted by $W_{k} \ldots$ Note that the multiplication sign refers to the $2 \mathrm{D}$ convolutional 
operator used to calculate the inner product of the filter model at each location in the input image. $f(\square)$ represents the nonlinear activation function, and $x_{k p q}$ denotes the element at location $(p, q)$ contained by the pooling region $\mathfrak{R}_{i j}$, which embodies a receptive field around the position $(i, j)$ [16]. The objective of image segmentation is to partition a given image into multiple regions (overlapping or not) that are considered meaningful according to some objective criterion or homogeneity in some feature space. This definition relies on selecting an objective function and depending on this criterion, the purpose of segmentation may change. In semantic segmentation, also known as scene labeling, we are interested in labeling all pixels in a given image. Each pixel should receive a semantic label (person) based on its surrounding information (context). The two most common semantic segmentation approaches are grammar-based and graphical models-based methods, which rely on Markov random fields or conditional random fields $[21,22]$.

\section{Automated ROI Detection using Deep CNN}

Many methods have been developed for digital pathology image analysis, ranging from rule-based to machine learning applications. Recently, a deep-dive, learning based approach has been shown to outperform traditional machine learning methods by automating end-to end processing in many image analysis tasks. In the medical imaging field deep CNN have been used successfully for various applications. For bone age prediction, existing deep-depth learning-base applications have shown potential for determine bone maturity. In this paper, we proposed method was executed on four NVIDA servers and the performance of each deep-CNN model was maintained. $20 \%$ of the data was used as a test set, and overfitting was applied to assess cross-propelling loss and accuracy of the testing and testing data. The losses used in the proposed method are calculated as follows (2).

$$
L=-(1 / n) \sum_{i=1}^{n} \log (P(\text { CorrectClass })
$$

The basic hyper parameter settings for deep running are as follows: learning rate $=$ 0.001 , regularization $=\mathrm{L} 2$, batch size $=512$ and 256 and drop out $=0.5 \%$. These parameter value were selected as parameters optimized for testing in our system. Note that L2 normalization was used to limit the square size of the kernel weight to prevent oversetting the data, while the drop out was used to limit the simultaneous adaptation of the unit to prevent overflow.

\subsection{Image Normalization}

With the proposed paper, we improved the speed of network training speed and normalization performance by reducing the movement of internal covariates through basification. We must get the X-ray image in place before we can extract the growth plate into the region of interest in order to reenact and increase the maturity of the bone. Since the size of the input images very considerably from hundreds to thousands of pixels, some images show black bones on a white background, and others appear on a black background prior to preprocessing to extract a white bone. In general, differences in the radiography of the test sets have the problem of not learning the special points correctly. Accordingly, it is important to increase the accuracy of the model by eliminating as much unwanted noise as possible in the pre-treatment pipeline, which is performed to standardize the image. We use bone maturity to determine the age of a goal so we can identify, partition, standardize, and maximize the inalterable characteristics of the CNN 
image in response to the preprocessing the prophetical features. Figure 2 shows the process of standardizing input images.

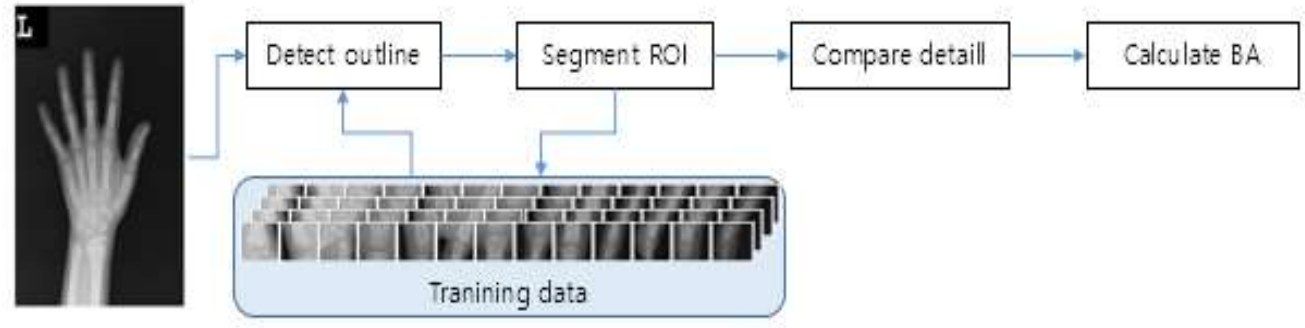

Figure 2. Conventional Calculation of Bone Age

The X-ray images used in this study were different sizes and were based on different grayscale; thus, image normalization was performed in the carry image standardization phase. First step, we calculated the average from each image using the $7 \times 7$ size image patch, and we performed grayscale normalization by comparing them to the absolute value at the resolution of the given image. This effectively determines whether the image has a white or black background, thereby making it possible to standardize all image to black background. In an $M_{w} \times N_{w}$ image, the neighboring window $w$ is represented as follows (3).

$$
\Omega_{w}=-\sum_{j=0}^{G-1} P_{j} \log _{2}\left(P_{j}\right) \quad \text { where } \quad P_{w}=n_{w} / M_{w} \times N_{w}
$$

Here, $P_{j}$ the probability of grayscale $j$ in the neighborhood $\Omega_{w}, n_{w}$ is the number of pixels with grayscale $j$ in $\Omega_{w}$. The second step is to standardize the size of the input image. Here we used left handed X-ray images generally have a rectangular shape; thus, the image were adjusted to 512 pixels high, then maintained at horizontal and vertical rate with zero-pattering to adjust the height of the image. Thus, images were standardized to a $512 \times 512$ pixels to optimize deep CNN performance and processing speed. An overly large image can improve the performance of the $\mathrm{CNN}$; however, large image require excessive processing time. In addition, overly small images reduce the preprocessing process of $\mathrm{CNN}$, so it is used as a learning dataset by selecting an image size $512 \times 512$ pixels. Figure 2 shows a network of $2 \times 2$ polling layers and one output plane, where low-resolution label planes are merged to form high-resolution labels. This merger is a simple copy operation that matches the pixels in a low-resolution label plane to the position of the original pixel in proportion to the number of polling layers in the previous month. The proposed algorithm is based on TW3. Automatic bone age calculation is performed using the CNN. There are five different types of objects in hand radiographs, i.e., bone, tissue, background, collimation, and annotation markers. To segment the hand and wrist from radiographs, we utilized the CNN to detect bones and tissues, constructed a hand/wrist mask, and applied a vision pipeline to standardize the images. 


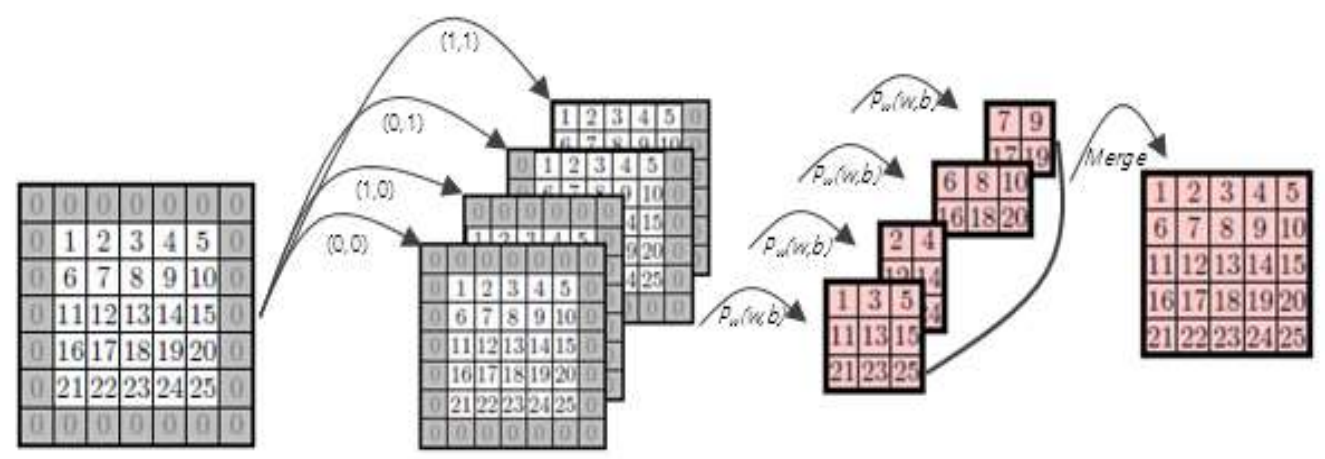

Figure 3. Polling Layers of Deep CNN

As shown in Figure 3, image patches for the five classes were sampled in the normalized images using ROIs. In the network, the image is input and the region of interest is output. The proposed method shows to output, one for the probability of a class in the ROI and the other for the class-specific boundary offset. The proposed model learns to optimize the two output layers using $p=\left(p_{0}, p_{1} . . p_{k}\right)$ and outputs the bounding box regression offset using $t^{k}=\left(t_{x}^{k}, t_{y}^{k}, t_{w}^{k}, t_{h}^{k}\right)$. Here, $t^{k}$ is parametrized such that it specifies a scale-invariant translation and log scale height /width shift relative to an object proposal. In addition, we extract an area of interest of $800 \times 800$ pixels and 1300 $\times 1300$ pixels form the original image, and we extract special points $(400 \times 400$ and $650 \times 650$ pixels) form the reduced image. In the experiment, we found that it is sufficient to determine the maturity of a bone using a reduced image.

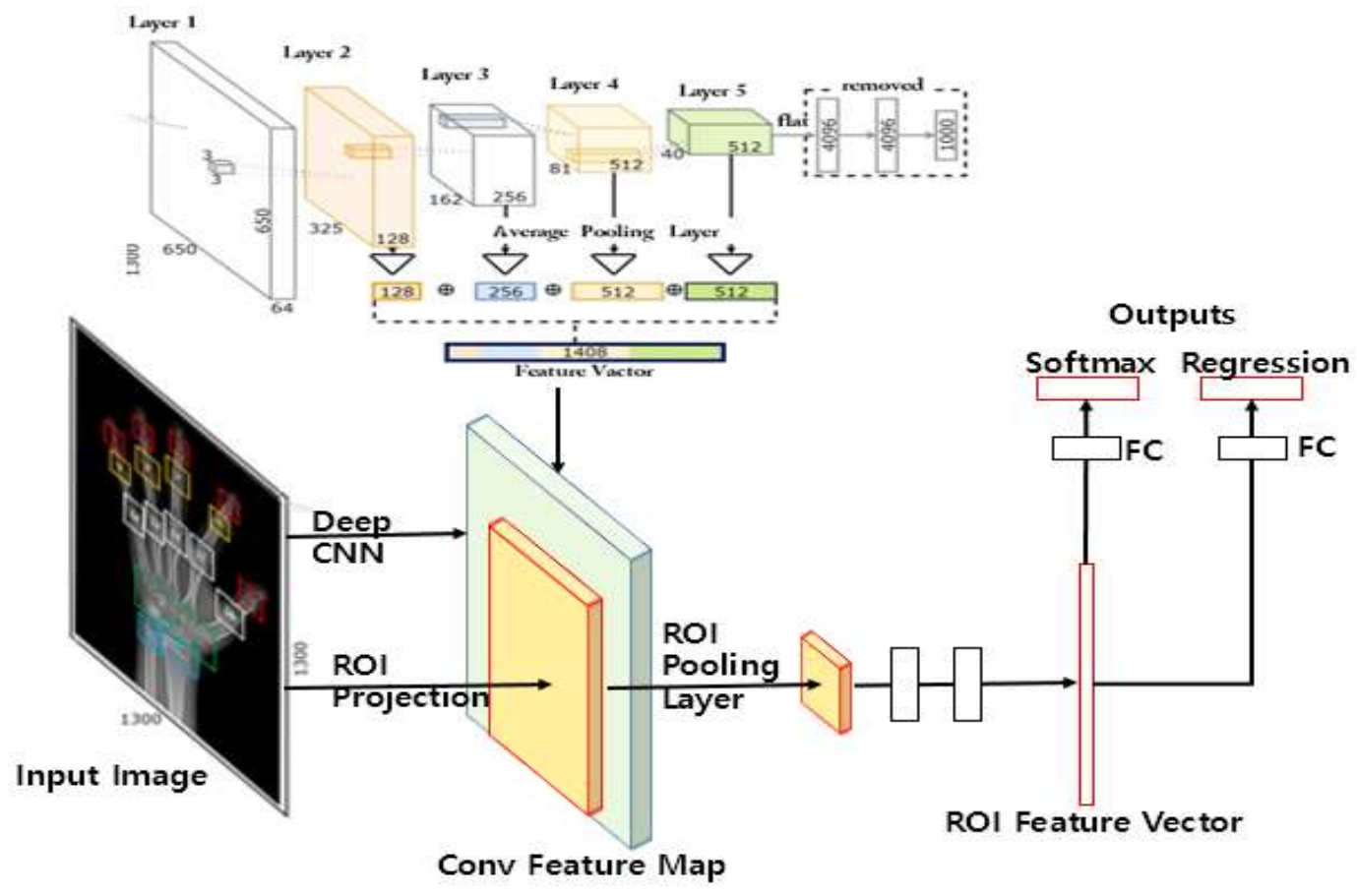

Figure 4. Our Proposed Network Extracts ROI Form Input Images based on CNN and RNN

Thus, the learning process of the proposed extracts and codes 20 special points for each image. Then the next set of 20 descriptors is combined into a single descriptor through 
three normal pooling. The Figure 4 show that our proposed network extracts ROI form input images based on CNN and RNN.

(4) shows the combination of the single descriptors through 3-normal pooling. Here, hyper parameter $\rho=3, N$ is the number of $f, d$ is the descriptor of the feature point, and $d$ image is the descriptor of the pooled image. In addition, the p-norm of the vector provides the maximum value for $\rho=1$ and $\rho \rightarrow \infty$. As a result, in each original image, we obtain 300 descriptors (50 ROI increase feature point $\times 2$ feature size $\times 3 \mathrm{CNN}$ encoder $=300$ )

$$
d_{p o o l}=\left(\frac{1}{N} \sum_{i=1}^{N}\left(d_{i}\right)^{p}\right)^{\frac{1}{p}}
$$

The sampled patch comprises a balanced set of data using on one million samples for each class. In the proposed method, a range of object patches is created using 1000 unique radiography images selected at randomly from the training dataset. Note that we used LeNet5 to learn the training data for deep CNN and learn gender recognition and specialty stores.

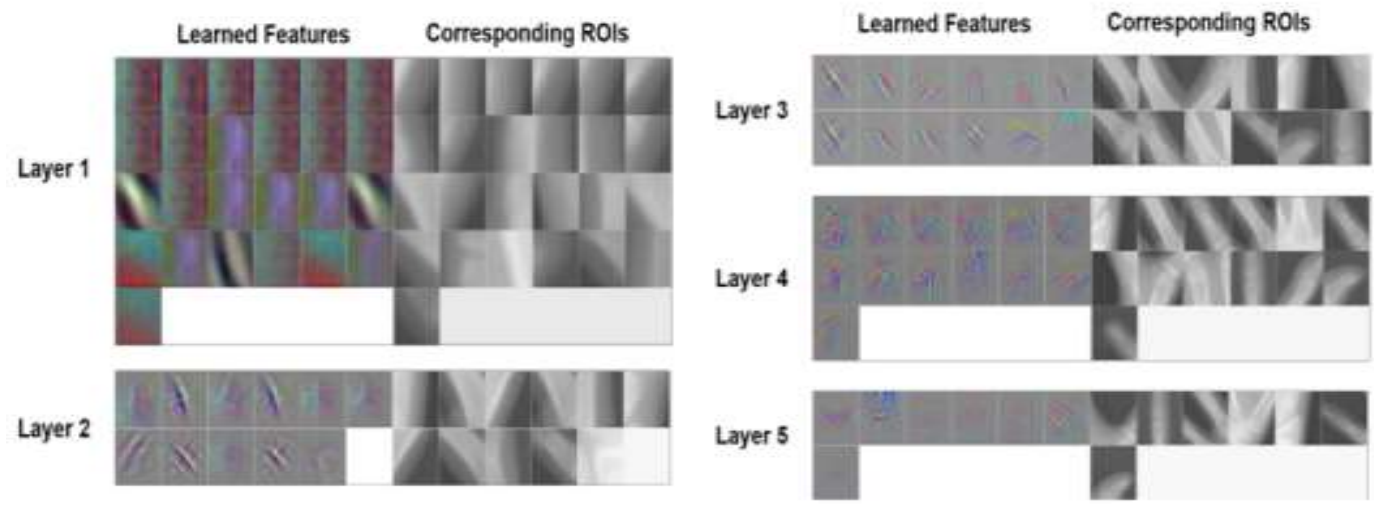

Figure 5. Deep Learned Features for each Layer and Corresponding ROls

Figure 5 show each layer's features are displayed in a different block. Within each block, we show a randomly chosen subset of features at epochs. Image contrast is artificially enhanced and the layer images are best viewed in electronic form. Occlude covers the image region that appears in the visualization. This Figure 5 shows that the visualization actually validates the image structure that stimulates the feature map, so it validates the other visualizations.

\section{Simulation and Result}

\subsection{Training Details}

Each image in the training dataset was normalized by padding it appropriately such that the mean and unit variation values were both zero. All networks randomly select form a set of training datasets, and if we randomly sample the patches surrounding the selected pixels, we will select our ROI. To evaluate the proposed method, two accuracy measurements were considered for the ROI images in the extraction area. The flowing Figure 6 shows special points used in learning by extracting features form the images used in training sets. 


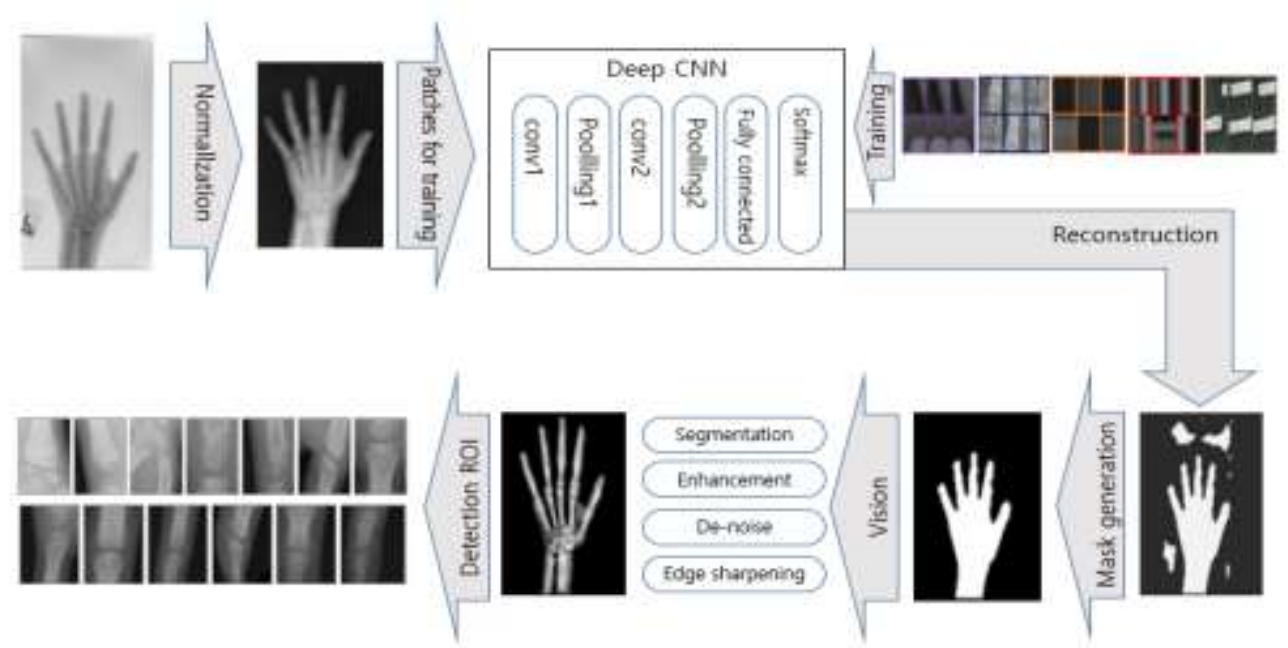

\section{Figure 6. Overview of a Deep CNN-based Preprocessing Engine to Automatically Detect a Hand Input Images}

The first is the accuracy per pixel of the training set image. This is the ratio of the pixels grouped together to ensure correct classification of the accuracy of the reference image considering the characteristics of the TW3 method. Second, we consider the average accuracy of the classes for each image in the training data set. As the growth plate differs relative to age, sex, and extraction image for each region, all classes have standardized this measurement by giving it the same weights.

We used data to simulate the algorithm (250 images). Here each image finds 14 ROIs and forwards the class to the return value. Each class (A, B, C, D, E, F, G and $\mathrm{H})$ has standardized values with the TW3 method, and the proposed algorithm produced precise ROI values using the CNN. To evaluate the proposed model, we measured the root mean squared error (RMSE) and mean accuracy (mAP) relative to ROI detection to determine bone maturity using the learning model. Table 11 shows the experiment results. We conducted a comparative experiment using existing taxonomy algorithms to evaluate the proposed method. Herein, we tested 250 left hand X-ray images (158 males and 92 female) to measure bone age. The results showed that male age was clearly divided between one year rather than SVMs, and female age was greater than 1.3 years. For females, the average of female over the age of $0.52 \%$ was determined in the experiment.

Table 1. Results of the Experiment

\begin{tabular}{|l|c|c|c|c|}
\hline \multirow{2}{*}{} & \multicolumn{2}{|c|}{ Male } & \multicolumn{2}{c|}{ Female } \\
\cline { 2 - 5 } & RMSE & mAP & RMSE & mAP \\
\hline SVM & 1.45 year & $32.4 \%$ & 1.89 year & $33.8 \%$ \\
\hline CNN & 0.83 year & $49.5 \%$ & 1.08 year & $47.5 \%$ \\
\hline Proposed & 0.71 year & $48.6 \%$ & 0.98 year & $48.3 \%$ \\
\hline
\end{tabular}

We also compared the sensitivity and accuracy of the proposed method to well-known classification algorithms for use a supplementary diagnostic program to determine bone maturity. Figure 7 shows the experimental results. 


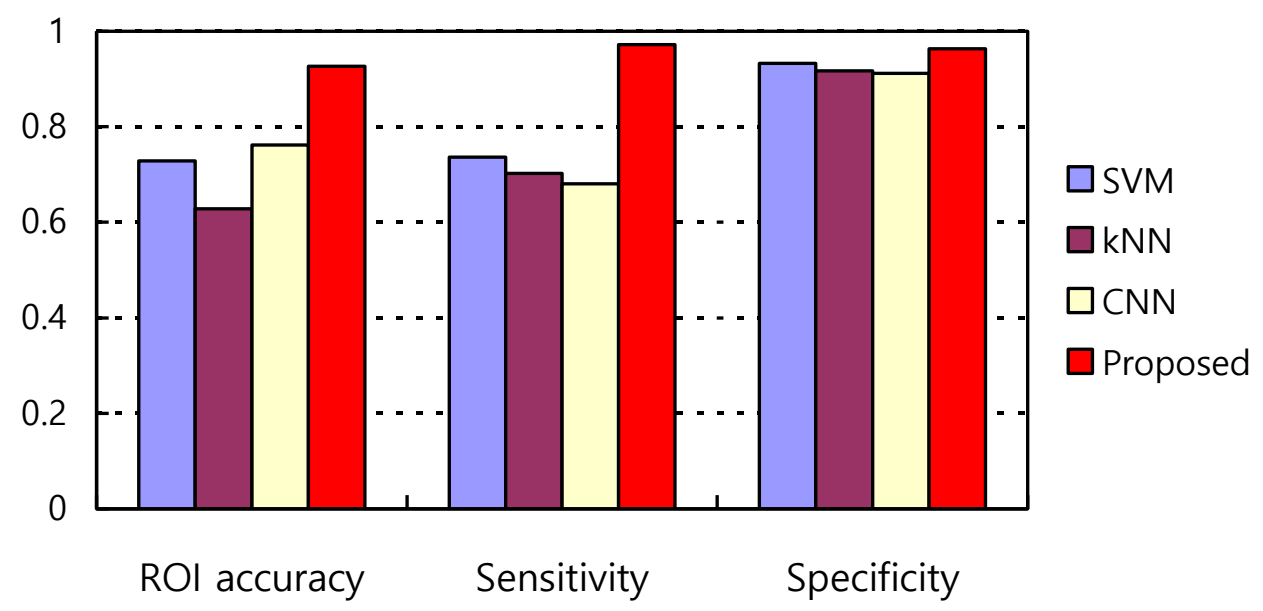

Figure 7. Results of the Experiment in the Proposed Model

The proposed model learned by increasing the amount of data into the CNN after applying automated preprocessing engines using sample of the original images. The learning outcomes were used to extract features and print the results from the input images. The results showed that $20 \%-40 \%$ of respondents had higher accuracy than the previous method of SVM, kNN and CNN. The replay rate of the input images was also high $(40 \%)$ and the specificity was also higher ( $96 \%$ or more).

\section{Conclusion and Future Work}

Recently, as the performance of medical imaging devices has improved, computer based image analysis has become important for medical diagnosis. Rapid and accurate segmentation of medical images is required to use medical images effectively; however, when the area to be divided is small, information used to determining the divided area is insufficient and may be removed in the dividing process. In this paper, we proposed an automatic bone age determination system using left-handed X-ray images. The proposed method automatically extracts an ROI from an input image using CNN- and RNN-based learning to improve accuracy. We also proposed a preprocessing learning algorithm and automatically classify ROIs a CNN and RNN, which are fine-tuned through using TW3based image standardization. The methodology has already been compared with the deeplearning algorithms proposed. The proposed paper proposed a multi-dimensional deeprunning algorithm to categorize the ROI extraction for bone maturity judgements and the bone maturity determination of the extracted images. The proposed algorithm automatically extracts the ROI for the input X-ray image based on a well-trained algorithm and generated bone maturity (RUS) values in the area extracted from the learning based. The RUS value represents the maturity of the bone and can automatically determine the age of the bone in the input image. Testing with the SVM, CNN and kNN algorithms to determine the performance of the proposed algorithm showed that the proposed system was at least $40 \%$ more sensitive. In this study, we have developed the Korean standard bone age TW3 reference model and demonstrated the applicability of the proposed algorithm as a CAD program by improving the performance of an automatic bone maturity classification system. 


\section{Acknowledgments}

This work was supported by the Starting growth Technological R\&D Program (S2537079) funded by the Small and Medium Business Administration (SMBA, Korea).

\section{References}

[1] M. J. Berst, L. Dolan, M. M. Bogdanowicz, M. A. Stevens, S, Chow and E. A. Brandser, "Effect of knowledge of chronologic age on the variability of pediatric bone age determined using the Greulich and Pyle standards", Journal of American journal of Roentgenology, vol. 176, no. 2, (2001), pp. 507-510.

[2] V. D. Sanctis, A. T. Soliman, S. D Maio and S. Bedair, "Are the new automated methods for bone age estimation advantageous over the manual approaches?", Pediatric Endocrinology Reviews, vol. 12, no. 2, (2014), pp. 277-282.

[3] L. M. Davis, B. J. Theobald and A. Bagnall, "Automated bone age assessment using feature extraction", Proceedings of the International Conference on Intelligent Data Engineering and Automated Learning, Berlin, Heidelberg, (2012) August 29-31.

[4] P. M. Garamendi, M. I. Landa, M. C. Botella and I. Alemán, "Forensic Age Estimation on Digital X-ray Images: Medial Epiphyses of the Clavicle and First Rib Ossification in Relation to Chronological Age", Journal of Forensic Sciences, vol. 56, no. S1, (2011), pp. 3-12.

[5] E. Pietka, A. Gertych, S. Pospiech, F. Cao, H. K. Huang and V. Gilsanz, "Computer-assisted bone age assessment: Image preprocessing and epiphyseal/metaphyseal ROI extraction", Journal of IEEE Transactions on Medical Imaging, vol. 20, no. 8, (2001), pp. 715-729.

[6] R. Zhu, R. Zhang and D. Xue, "Lesion detection of endoscopy images based on convolutional neural network features", Processings of the Image and signal, Shenyang, China, (2016) October 14-16.

[7] S. Y. Kim, Y. J. Oh, J. Y. Shin, Y. J. Rhie and K. H. Lee, "Comparison of the Greulich-Pyle and Tanner Whitehouse (TW3) methods in bone age assessment", Journal of Korean Society of Pediatric Endocrinology, vol. 13, no. 1, (2008), pp. 50-55.

[8] F. Torres, M. A. Bravo, E. Salinas, G. Triana and P. Arbeláez, "Bone age detection via carpogram analysis using convolutional neural networks", Proceedings of the 13th International Conference on Medical Information Processing and Analysis, San Andres Island, Colombia, (2017) November 17.

[9] C. Spampinato, S. Palazzo, D. Giordano, M. Aldinucci and R. Leonardi, "Deep learning for automated skeletal bone age assessment in X-ray images", Journal of Medical Image Analysis, vol. 36, (2017), pp. 41-51.

[10] R. M. Malina, M. J. Coelho-e-Silva, A. J. Figueiredo, R. M. Philippaerts, N. Hirose, M. E. P. Reyes and L.F. Guglielmo, "Tanner-Whitehouse Skeletal Ages in Male Youth Soccer Players: TW2 or TW3?", Journal of Sports Medicine, vol. 48, no. 4, (2018), pp. 1-18.

[11] Z. Yan, Y. Zhan, S. Zhang, D. Metaxas and X. Zhou, "Multi-Instance Multi-Stage Deep Learning for Medical Image Recognition”, Deep Learning for Medical Image Analysis publishers, Elsevier, (2017).

[12] J. Zhou, Z. Li, W. Zhi, B. Liang, D. Moses and L. I. Dawes, "Using Convolutional Neural Networks and Transfer Learning for Bone Age Classification", Processing's of the International Conference on Digital Image Computing: Techniques and Applications, Sydney, Australia, (2017) November 29-December 1.

[13] X. Peng and C. Schmid, "Multi-region two-stream R-CNN for action detection", Proceedings of the $14^{\text {th }}$ European Conference on Computer Vision. Springer (LNCS, vol 9908), Amsterdam, the Netherlands (2016) October 8-16.

[14] M. Mansourvar, A. Asemi, R. G. Raj, S. A. Kareem, C. D. Antony, N. Idris and M. S. Baba, "A fuzzy Inference System for Skeletal Age Assessment in Living Individual", International Journal of Fuzzy Systems, vol. 19, no. 3, (2017), pp. 838-848.

[15] S. Simu, S. Lal, K. Fadte and A. Harlapur, "Fully automatic segmentation of phalanges from hand radiographs for bone age assessment", Journal of Computer Methods in Biomechanics and Bio Medical Engineering: Imaging \& Visualization, (2017), pp. 1-26.

[16] Y. B. Cho, S. H. Woo and C. S. Han, "An iris recognition algorithm using weighted Hough transform and PCA", Proceedings of the 2017 International Conference on Future Information \& Communication Engineering, Irkutsk, Russian Federation, (2017) June 27-30.

[17] J. Kotchakorn, A. I. Ngamnij and A. I. Somjit, "Ontology Mapping and Rule-Based Inference for Learning Resource Integration", Journal of information and communication convergence engineering, vol. 14, no. 2, (2016), pp. 97-105.

[18] S. W. Shin, K. S. Kim, S. M. Lee and C.G. Song, "Image Enhancement with Rotating Kernel Transformation Filter Generated by Bresenham's Algorithm", International Journal of Korean Institute of Electrical Engineers, vol. 61, no. 6, (2012), pp. 872-878.

[19] Y. B. Cho and S. H. Woo, "The Kirsch-Laplacian Edge Detection Algorithm for Predicting Iris-based Desease", Proceedings of the 2017 IEEE 21st International Conference on Computer Supported Cooperative Work in Design, Wellington, NewZealand, (2017) April 26-28. 
[20] S. Ren, K. He, R. Girshick and J. Sun, "Faster R-CNN: towards real-time object detection with region proposal networks", Journal of the IEEE Transactions on Pattern Analysis and Machine Intelligence, vol. 39, no. 6, (2017), pp. 1137-1149.

[21] M. C. Lee, K. Inoue and M. Cho, "Three-Dimensional Automatic Target Recognition System Based on Optical Integral Imaging Reconstruction”, Journal of Information and Communication Convergence Engineering, vol. 14, no. 1, (2016), pp 51-56.

[22] B.B. Ehteshami, J. Linz, B. Glass, M. Mullooly, G. L. Gierach, M. E. Sherman and A. H. Beck, "Deep learning-based assessment of tumor-associated stroma for diagnosing breast cancer in histopathology images", Proceedings of the $14^{\text {th }}$ international symposium on biomedical imageing, Melbourne, Australia, (2017) April 18-27.

[23] K. Inoue, M. C. Lee, C. S. Kim and M.J. Cho, "Color Compensation of an Underwater Imaging System Using Electromagnetic Wave Propagation", Journal of information and communication convergence engineering, vol. 14, no. 3, (2016), pp. 200-206.

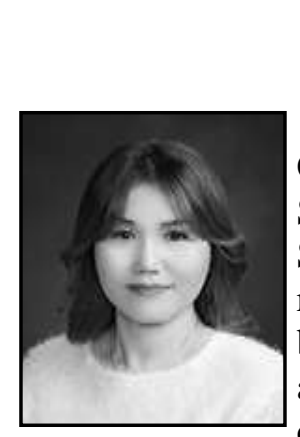

\section{Authors}

Youngbok Cho, is an Associate Professor at the Department of Computer \& Information Security, Daejeon University. Daejeon, South Korea. She received her M.S. and Ph.D. degrees in Computer Science from Chungbuk National University, in 2006 and 2012 respectively. She has been involved in few national research and has been expert adviser of SME in Korea to follow up research projects and evaluator of Korea R\&D a national research proposals. She is currently working in the department of computer \& information security, Daejeon University. Her research interest includes also include network security, medical information security and medical image processing.

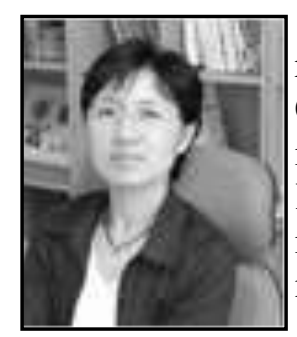

Sunghee Woo, was born in Chungbuk, Korea in 1968. She received M.S.and Ph.D. from department of Computer Science, Chungbuk National University, in 1993 and 1999 respectively. She is currently working in the department of Medical IT Engineering, Korea National University of Transportation. Her major field of study is network security, medical informatics, healthcare, and computer network. 
International Journal of Grid and Distributed Computing

Vol. 11, No. 7 (2018) 